\title{
CFA Based Simultaneous Multispectral Imaging and Illuminant Estimation
}

\author{
Raju Shrestha and Jon Yngve Hardeberg \\ The Norwegian Colour and Visual Computing Laboratory, \\ Gjøvik University College, Gjøvik, Norway \\ raju.shrestha@hig.no \\ http://www . colourlab.no
}

\begin{abstract}
This paper proposes an extension to the CFA based multispectral imaging with an added capability of illuminant estimation. A special filter is used on top of regular R, G and B filters of a camera, replacing one of the two green filters, with one of them. This gives a six channel multispectral image. A normal RGB image is produced by the RGB filters. The corresponding filtered RGB image is obtained using the filtered RGB channels. The two images of a scene allow estimating the illuminant using the chromagenic illuminant estimation algorithm. The proposed system is thus capable of acquiring not only multispectral image but also normal RGB image, and at the same time capable of estimating the illuminant under which the image is captured. This makes the system useful in many applications in color imaging and computer vision. Simulation experiments confirm the effectiveness of the proposed system.
\end{abstract}

Keywords: multispectral, color constancy, illuminant estimation, chromagenic, color filter array, cfa, mcfa.

\section{Introduction}

Conventional three channel (usually RGB) color imaging suffers from limitations like metamerism and environment dependency. Multispectral imaging provides a solution to these limitations. However, most of the state-of-the art multispectral imaging systems acquire images in multiple shots [17, 20, 35, 36, and this limits their use only to static scenes. Multispectral color filter array (MCFA) [2 6 23, 233] is one of the promising one-shot solutions which is of our interest in this paper. The most commonly used color filter array (CFA) in commercial digital cameras is the 3-channel Bayer pattern 4]. MCFA extends this for multispectral imaging with the use of more than three channels. Farrell et al. [10] suggested the use of an additional color filter to generate 6 -component color formation. The number and type of filters, their spatial arrangement and demosaicking algorithm are three important issues involved in a MCFA based multispectral camera. A number of works have been done in the field of filter selection for multispectral acquisition systems, e.g. the work by Hardeberg et al. [18]. Miao et al. 25] proposed a

S. Tominaga, R. Schettini, and A. Trémeau (Eds.): CCIW 2013, LNCS 7786, pp. 158-170, 2013.

(C) Springer-Verlag Berlin Heidelberg 2013 
spatial arrangement of MCFA filters, based on the probability of appearance of the corresponding band. Shrestha et al. 33. investigated the influence of spatial arrangements on the performance of the systems. There are many demosaicking algorithms, mostly proposed for 3-channel images, however these can mostly be extended to MCFA with little modification. Miao et al. 26] proposed a binary tree-based generic demosaicking algorithm for MCFAs.

Color constancy is yet another important issue in color imaging. It is the ability to account for the color of the light source which allows seeing the color of an object more or less the same under different lighting conditions. Since human vision has this ability, it is said to be color constant [1,5]. Computational color constancy tries to emulate the color constancy in color imaging, and this is one of the fundamental requirements in many color imaging and computer vision applications. Computational color constancy models, in general, comprise of two steps: illuminant estimation and color correction. The illuminant estimation is the primary task in a computational color constancy algorithm. Knowing the estimated illuminant, the effects of the color of the illuminant are corrected to obtain the desired color constant image. Many methods have been proposed for the illuminant estimation. Some example methods are gray-world [7], max-RGB [22], a gamut based algorithm [14, neural networks [8], color-by-correlation [11] and a Bayesian method [16]. Yet another color constancy algorithm, known as the chromagenic color constancy, has been proposed by Finlayson et al. 12] which uses a special color filter which they named as chromagenic. This algorithm estimates the illuminant from two images: a normal RGB and a filtered RGB, of a scene. The algorithm has registration problems and also requires two shots of an image. Fredembach and Finlayson [15] proposed the bright-chromagenic algorithm aiming to remedy the large error problem and to relax the registration constraint to some extent. However, the two shot requirement still severely limits its applicability to static scenes only. We proposed a one shot solution to the chromagenic color constancy using a stereo camera [30, and combined with the multispectral imaging capability [29,34] for both the multispectral imaging and the color constancy 32 . Stereo based approach still has occlusion and registration issues to be dealt with. CFA based approach is a solution for this. We proposed a computational color constancy approach using chromagenic filters in CFAs which is also a one shot solution but without any registration issue [31. In this case, the bright-chromagenic algorithm does not perform significantly different from the original chromagenic algorithm.

In this paper, we extend the CFA based computational color constancy [31] a step further with the added capability for the multispectral imaging. This system has the same capabilities as the stereo based system [32, but with a different approach using the CFA, that does not have any registration issue. The system is capable of acquiring multispectral image, and at the same time estimating the illuminant under which the image has been acquired. Chromagenic filters are introduced into the CFA itself. We replace one of the two green filters in a typical Bayer pattern 4 by a combination of chromagenic and $R, G, B$ filters. This allows obtaining two images of the same scene via demosaicking, a normal 
RGB image, and a chromagenic image, equivalent to the RGB image through a chromagenic filter. The illuminant can then be estimated using the chromagenic algorithm proposed by Finlayson et al. [12. Moreover, combining the normal and the filtered RGBs of a scene allows obtaining a 6-channel multispectral image of the scene. Either custom designed chromagenic filter or an appropriate filter selected from a given set that minimizes the spectral/color estimation error and the illuminant estimation error, is used. Experiments with a simulated system confirm the effectiveness of the proposed system. The paper outlines how chromagenic color constancy and the MCFA based multispectral imaging technique can be combined to have both the capabilities in a single system which could be useful in many color imaging and computer vision applications.

After this introduction, we present next the proposed system along with the multispectral imaging and the illuminant estimation methods. We then present the experiments and results. The results will be discussed next, and finally we conclude the paper.

\section{Proposed System}

We proposed a CFA based computational color constancy in 31. The proposed system, in this paper, uses the same CFA design, but extends the capability of the system for the multispectral imaging as well. The chromagenic filters are placed on top of $R, G$ and $B$ filters, and the resulting filters denoted as $R^{F}$, $G^{F}$ or $B^{F}$ respectively are used in place of one of the green filters in a typical Bayer pattern 4]. To somewhat compensate for the loss of a green filter, we use two $G^{F}$ filters for one $R^{F}$ and one $B^{F}$ filters resulting the chromagenic filter pattern of $R^{F} G^{F} G^{F} B^{F}$. This pattern also follows the two important CFA design requirements: the spectral consistency and the spatial uniformity [25]. To present the same reconstruction performance throughout the image plane, the spectral consistency requires that pixels should always have the same number of neighbors of a certain spectral band within a neighborhood of certain distance. The spatial uniformity requires that the filter array for each spectral band samples the entire image as evenly as possible. Figure 1(a) illustrates a Bayer CFA pattern in RGGB and Figure 1(b) illustrates the corresponding CFA pattern after the introduction of the chromagenic filters. Gray blocks in these figures shows the basic block which would be repeated uniformly in the whole image.

This CFA design allows obtaining two images of the same scene through demosaicking: a normal RGB image, and a chromagenic filtered image. An unfiltered image is obtained from the pixels with $R, G$ and $B$ filters, using a demosaicking algorithm. Similarly a filtered image is obtained from pixels with the $R^{F}, G^{F}$ and $B^{F}$ filters. An appropriate demosaicking algorithm can be used. A larger kernel might be required for the demosaicking of a filtered image, as can be seen with bigger gray block in Figure 1(b), and it might reduce some spatial resolution. Having two images: an unfiltered and a filtered, the illuminant can then be estimated using the chromagenic algorithm [12]. 


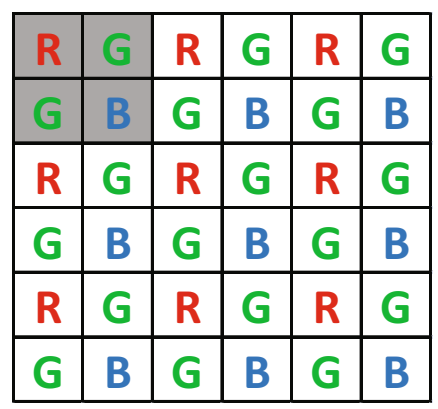

(a) Bayer CFA

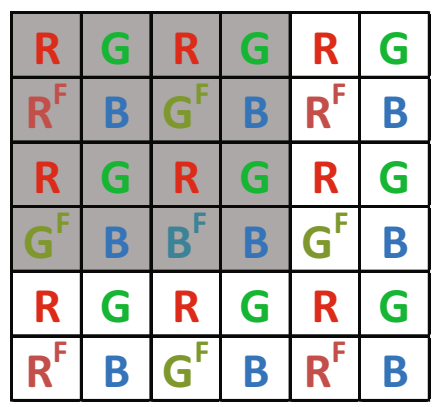

(b) Chromagenic CFA

Fig. 1. Illustration of CFAs with and without the chromagenic filters

In order to extend the system's capability to acquire multispectral image at the same time, a special chromagenic filter is used which effectively produces 6-channel multispectral image, by combining $R G B$ and $R^{F} G^{F} B^{F}$ channels. The filter can either be custom designed, or be selected from a set of filters through an exhaustive search. Since only one filter needs to be selected, the computational complexity for the exhaustive search is just $\mathcal{O}(n)$. A filter that produces minimum estimation errors with regards to both (within a given threshold) the multispectral output and the color constancy output, is selected. More explanation on this can be found in Section 3.2. We use here in this paper the minimum color estimation error as the criteria for the multispectral output for accurate color reproduction, and use the most widely used median angular error 3.19. for accurate illuminant estimation. However, depending on the application, spectral estimation error criteria could also be used.

In the subsections below, we present the multispectral system model and the illuminant estimation with the proposed system.

\subsection{Multispectral System Model}

In order to model the proposed multispectral system, the system can be considered to be comprised of two cameras: a normal RGB camera and a filtered RGB camera. Let $S=\left[s_{R}, s_{G}, s_{B}\right]$ be the matrix of spectral sensitivities of the three channels of the normal RGB camera, and similarly $S^{F}=\left[s_{R}^{F}, s_{G}^{F}, s_{B}^{F}\right]$ the matrix of the spectral sensitivities of the three channels of the filtered RGB camera. Let $L$ be the spectral power distribution of the light source, and $R$ the spectral reflectance of the surface captured by the camera. Let $n$ and $n^{F}$ be the noise vectors corresponding to the acquisition noise in the three channels of the normal and the filtered RGB cameras respectively. The camera responses of the normal and the filtered cameras: $C^{N}$ and $C^{F}$ are respectively given by:

$$
\begin{aligned}
C^{N} & =S^{\prime} \operatorname{Diag}(L) R+n \\
\text { and } C^{F} & =\left(S^{F}\right)^{\prime} \operatorname{Diag}(L) R+n^{F},
\end{aligned}
$$


where $S^{\prime}$ denotes the transpose of the matrix $S$. The combined response $C=$ $\left[\begin{array}{l}C^{N} \\ C^{F}\end{array}\right]$ of the two cameras gives six responses. The estimated spectral reflectance $(\tilde{R})$ is then obtained for the corresponding original reflectance $(R)$, from the camera response $C$ using a spectral estimation method. Among several widely used spectral estimation methods like Imai and Berns (IB) [21, Linear Regression, Polynomial (PN) [9] and Neural Network (NN) [24], we have used the linear regression method as it is simple, fast and produces good results [29, 34. Let $C_{\text {train }}$ and $C$ denote the camera responses of the training $\left(R_{\text {train }}\right)$ and the test $(R)$ targets respectively. Then, the estimated reflectance with the linear regression method is given by the following equation:

$$
\tilde{R}=R_{\text {train }} C_{\text {train }}^{+} C,
$$

where, $C^{+}$denotes the pseudo-inverse of $C$.

The estimated reflectances are evaluated using a spectral as well as a colorimetric metrics. PSNR (Peak-Signal-to-Noise-Ratio) has been used as the spectral metric, and $\Delta E_{a b}^{*}$ (CIELAB color difference) as the colorimetric metric. $P S N R$ is calculated as $20 \log _{10} \frac{1}{R M S E}$, where $R M S E$ is the root mean square error.

\subsection{Illuminant Estimation}

The two images of a scene allow estimating the illuminant as well using the chromagenic illuminant estimation method proposed by Finlayson et al. [12. Shrestha 31] has proposed chromagenic based illuminant estimation technique using CFA. Since the system is linear, the normal and the filtered camera responses can be related by the following equation:

$$
C^{F}=M C^{N}
$$

where $M$ is a $3 \times 3$ linear transformation matrix. $M$ depends on both the illuminant and the filter used, and it can be computed as:

$$
M=C^{F}\left(C^{N}\right)^{+} .
$$

The transformation matrix $M$ can be described as the transform that maps, in a least square sense, the unfiltered to the filtered responses of the camera under a given illuminant. The chromagenic illuminant estimation method is based on the assumption that we know all possible illuminants a priori. Let $l_{i}(\lambda), i=$ $1, \ldots, m$ be the spectral power distributions of the possible known illuminants, and $r_{j}(\lambda), i=1, \ldots, n$ the reflectances of the $n$ representative real world surfaces. For each illuminant $i$, we determine the camera responses without and with the chromagenic filter: $C_{i}^{N}$, and $C_{i}^{F}$ respectively, which are $3 \times n$ matrices whose $j^{\text {th }}$ column contains the camera responses of the $j^{\text {th }}$ surface under the $i^{\text {th }}$ illuminant. The transformation matrix $M_{i}$ for the $i^{\text {th }}$ illuminant $\left(l_{i}\right)$ is obtained using Eq. 5 . 
For a given test illuminant, we select an illuminant $l_{\text {est }}(\lambda)$ from all plausible illuminants $l_{i}$ as the estimated illuminant, which produces the minimum error:

$$
\text { est }=\underset{i}{\operatorname{argmin}}\left(e_{i}\right), i=1, \ldots, m
$$

where $e_{i}$ is the fitting error which can be calculated as:

$$
e_{i}=\left\|M_{i} C^{N}-C^{F}\right\|, i=1, \ldots, m .
$$

The illuminant estimation algorithm is evaluated using the framework proposed by Hordley and Finlayson [19], and using median angular error. The angular error is intensity independent, and it has been widely used in the literature 3,19. Let $C_{l_{\text {est }}}$ and $C_{l_{\text {act }}}$ be the camera responses of a white reflectance under the estimated and the actual illuminant respectively, then the angular error $e_{\text {ang }}$ is calculated as:

$$
e_{\text {ang }}=\cos ^{-1}\left(\frac{C_{l_{\mathrm{act}}}^{T} C_{l_{\mathrm{est}}}}{\left\|C_{l_{\mathrm{act}}}\right\|\left\|C_{l_{\mathrm{est}}}\right\|}\right)
$$

\section{$3 \quad$ Experiments}

In this section, we discuss the experimental setup and then present three different experiments and the results they produced.

\subsection{Experimental Setup}

All the experiments are carried out through simulations. The experimental setup, therefore, comprises of spectral data of a camera, filters, illuminants and surface reflectances. A Sony DXC930 camera whose spectral sensitivities data given by Barnard et al. [3] is used. The CIE D65 illuminant, and the CIE $196410^{\circ}$ color matching functions are used for the color computation. The linear regression spectral estimation method, and the two evaluation metrics discussed above are used for estimating the multispectral images and evaluating the multispectral imaging system.

For the illuminant estimation, the surface reflectances (denoted as $R$ ) from 1995 Munsell patches, the 87 measured training illuminants $\left(L_{87}\right)$, and the 287 test illuminants $\left(L_{287}\right)$; all the data from Barnard et al. 3] have been used. 265 optical filters from Omega Optical Inc., whose transmittances are available in the company website 28] are used.

In order to evaluate the system, hyperspectral images (illuminant-discarded) of the eight natural scenes from Nascimento et al. 27] have been used to acquire simulated images. Figure 2 shows the RGB images generated from these hyperspectral images. A simple bilinear interpolation is used for the demosaicking, and the results from the interpolated images are compared with the ideal system which doesn't require interpolation. The process of bilinear interpolation has been well described by Baone and Qi [2]. If we obtain an improved performance from the proposed system using a simple bilinear interpolation, we can expect much better results with a more sophisticated demosaicking algorithm. 


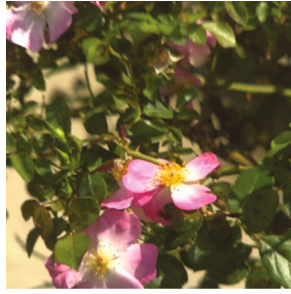

Scene \#1

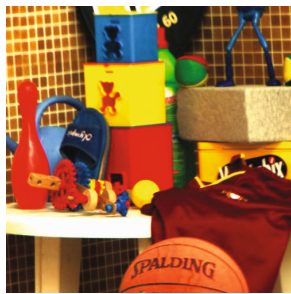

Scene \#5

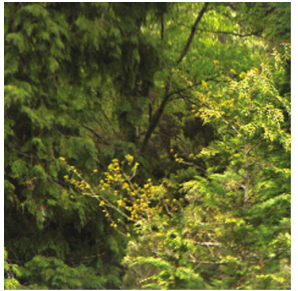

Scene \#2

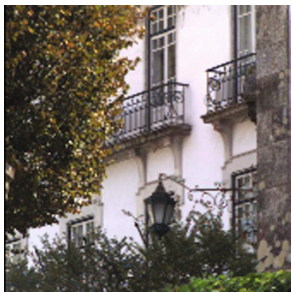

Scene \#6

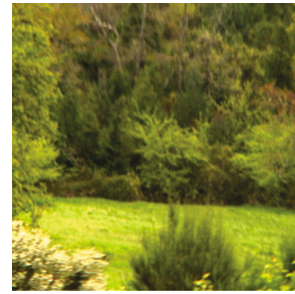

Scene \#3

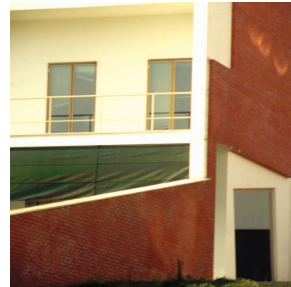

Scene \#7

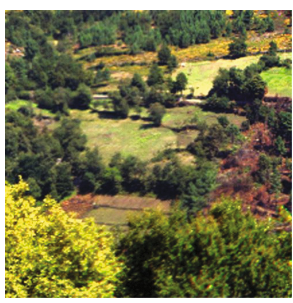

Scene \#4

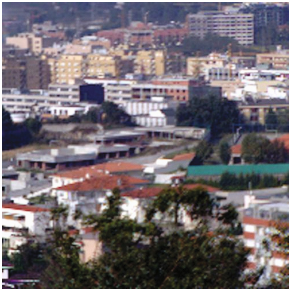

Scene \#8

Fig. 2. The RGB images obtained from the hyperspectral images of the 8 natural scenes from Nascimento et al. 27.

\subsection{Experiment I: Filter Selection}

Filters that produce the minimum $\Delta E_{a b}^{*}$ by the multispectral system, and the minimum illuminant estimation $\left(e_{a n g}\right)$ error, that lie within an acceptable error threshold values is considered acceptable. In other words, the system is optimized for the accuracy of the color reproduction and the illuminant estimation. A simple filter selection approach could be to plot the estimation errors obtained with all the filters used, with the angular error along the one axis and the color error along the other axis, and select a filter that produces minimum errors within the defined threshold range (Figure 3). A 10-fold cross validation method is used to select the training and the test targets from the 1995 Munsell surface reflectance data. Simulated images are generated from the hyperspectral data, under the illuminant D65 using the proposed MCFA based camera.

For the illuminant estimation, the transformation matrices $M_{i}, i=1 \ldots 87$ are computed by imaging the whole surface reflectances $R$, under the training illuminants $L_{87}$, and they are then used to estimate the test illuminants $L_{287}$. The test illuminants are estimated using the chromagenic algorithm.

One filter is selected from a set of filters through an exhaustive search. If there is a considerably large number of filters, computational complexity with the exhaustive search could be improved by introducing a secondary criterion which excludes all infeasible filters from costly computations. One such criterion could be that the filters which result in a maximum transmission factor of less than forty percent and less than ten percent of the maximum transmission factor in one or more channels are excluded [33, 34. We also skip those filters which produce high estimation errors (more than $10 \Delta E_{a b}^{*}$ or $8 e_{a n g}$ ). 


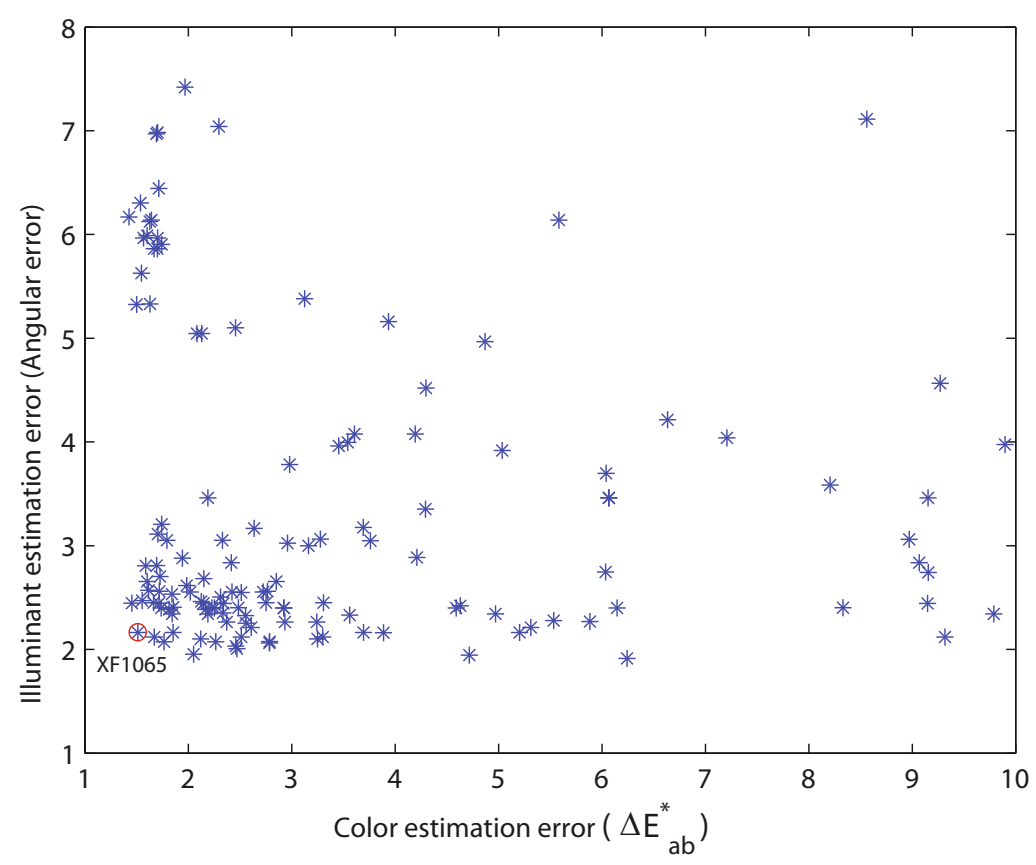

Fig. 3. Selection of a filter (red circled) from a set of 265 Omega filters

Depending on the application, an appropriate threshold values can be set for the color and the illuminant estimation errors. Here, as an illustration, we have chosen the $\Delta E_{a b}^{*}<1.6$ and $e_{a n g}<2.3^{\circ}$ as the threshold values. Fig. 3 shows the $\mathrm{XY}$ plot of the estimation errors produced by the 265 Omega filters, with the color estimation error along the $\mathrm{X}$-axis and the angular error along the $\mathrm{Y}$-axis. The filter selection algorithm chooses the XF1065 filter as shown in the figure. Fig. 4 shows the transmittance of the filter. This filter is then used in the next two experiments for the multispectral imaging and the illuminant estimation.

\subsection{Experiment II: Multispectral Imaging}

This experiment evaluates the proposed MCFA based multispectral system constructed from the $R G B$ filters of the Sony DXC930 camera, and $R^{F} G^{F} B^{F}$ filters obtained by combining the Omega XF1065 filter selected in the Experiment I with the $R G B$ filters. Fig. [5] shows the resulting normalized spectral sensitivities of the 6-channel multispectral imaging system. The six channel multispectral images are obtained from the normal and the filtered RGB camera responses of the 8 scenes, using the linear regression (LR) method. The performance of the system is then evaluated using the two evaluation metrics (RMS \& $\Delta E_{a b}^{*}$ ). 
Table 1 shows the average mean estimation errors produced by the proposed 6-channel multispectral system along with the errors produced by the classical 3 -channel camera, for all the eight images. The results show that the proposed 6-channel system produces the average $\Delta E_{a b}^{*}$ and $P S N R$ of 1.8 and 33.68 respectively, while the 3 -channel system produces the corresponding values of 2.43 and 32.40. Similarly, the ideal (which does not require interpolation) 6-channel multispectral system produces the metric values of 0.65 and 34.51 , while the 3 -channel system produces the metric values of 1.87 and 33.67.

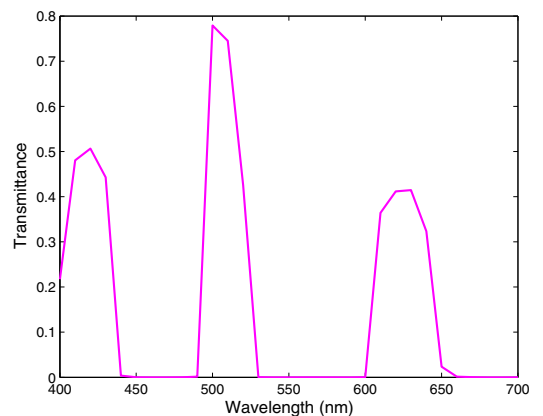

Fig. 4. Transmittance of the Omega XF1065 filter

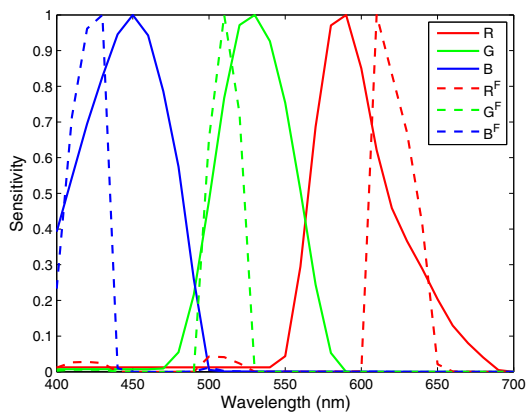

Fig. 5. Normalized spectral sensitivities of the 6-channel multispectral system

Table 1. Average spectral and color estimation errors for the 8 scenes

\begin{tabular}{|c|c|c|c|c|c|c|c|c|}
\hline \multirow{2}{*}{ Scene \# } & \multicolumn{4}{|c|}{ Without Interploation } & \multicolumn{3}{c|}{ With CFA/MCFA Interpolation } \\
\cline { 2 - 9 } & 3-chan. system & 6-chan. system & \multicolumn{3}{|c|}{ 3-chan. system } & 6-chan. system \\
\cline { 2 - 9 } & PSNR & $\Delta \mathrm{E}^{*}{ }_{\mathrm{ab}}$ & PSNR & $\Delta \mathrm{E}^{*}{ }_{\mathrm{ab}}$ & PSNR & $\Delta \mathrm{E}^{*}{ }_{\mathrm{ab}}$ & PSNR & $\Delta \mathrm{E}^{*}{ }_{\mathrm{ab}}$ \\
\hline $\mathbf{1}$ & 28.95 & 1.50 & 29.48 & 0.60 & 28.84 & 2.27 & 29.78 & 1.81 \\
\hline $\mathbf{2}$ & 33.58 & 1.42 & 33.70 & 0.78 & 33.49 & 2.53 & 33.41 & 2.25 \\
\hline $\mathbf{3}$ & 22.28 & 1.86 & 22.59 & 0.53 & 22.28 & 2.12 & 22.53 & 1.22 \\
\hline $\mathbf{4}$ & 35.48 & 1.17 & 35.47 & 0.41 & 35.43 & 1.54 & 35.86 & 1.03 \\
\hline $\mathbf{5}$ & 23.60 & 3.20 & 25.57 & 1.44 & 23.46 & 4.11 & 24.54 & 2.78 \\
\hline $\mathbf{6}$ & 48.01 & 1.33 & 51.07 & 0.43 & 47.70 & 1.94 & 51.94 & 1.05 \\
\hline $\mathbf{7}$ & 41.85 & 1.10 & 42.14 & 0.42 & 41.61 & 1.57 & 43.14 & 0.92 \\
\hline $\mathbf{8}$ & 35.63 & 3.35 & 36.03 & 0.55 & 26.41 & 3.35 & 28.27 & 3.31 \\
\hline Average & $\mathbf{3 3 . 6 7}$ & $\mathbf{1 . 8 7}$ & $\mathbf{3 4 . 5 1}$ & $\mathbf{0 . 6 5}$ & $\mathbf{3 2 . 4 0}$ & $\mathbf{2 . 4 3}$ & $\mathbf{3 3 . 6 8}$ & $\mathbf{1 . 8 0}$ \\
\hline
\end{tabular}




\subsection{Experiment III: Illuminant Estimation}

In this experiment, we obtain the unfiltered and the filtered versions of each image from the hyperspectral images for every test illuminant $L_{287}$. The test illuminant is then estimated for each scene using the chromagenic algorithm. The median angular errors produced by the chromagenic algorithm along with the gray world and the max-RGB methods are given in Table 2 The results show that the proposed system produces the median (from 8 scenes) of the median angular errors of 5.05, 6.58 and 9.55 with these algorithms respectively. The ideal system which doesn't require interpolation produces the corresponding values of $4.90,6.62$ and 9.46 .

Table 2. Median angular errors for the 8 scenes

\begin{tabular}{|c|c|c|c|c|c|c|}
\hline \multirow{2}{*}{ Scene \# } & \multicolumn{3}{|c|}{ Without interpolation } & \multicolumn{3}{c|}{ With CFA Interpolation } \\
\cline { 2 - 8 } & Gray & Max & Chroma- & Gray & Max & Chroma- \\
& World & RGB & genic & World & RGB & genic \\
\hline $\mathbf{1}$ & 12.45 & 8.34 & 4.95 & 13.94 & 8.59 & 5.20 \\
\hline $\mathbf{2}$ & 11.68 & 7.50 & 5.88 & 11.65 & 7.40 & 6.18 \\
\hline $\mathbf{3}$ & 12.61 & 7.17 & 7.06 & 12.67 & 13.50 & 7.06 \\
\hline $\mathbf{4}$ & 7.25 & 8.86 & 4.86 & 7.46 & 9.68 & 4.90 \\
\hline $\mathbf{5}$ & 12.18 & 2.40 & 2.44 & 13.01 & 2.28 & 2.61 \\
\hline $\mathbf{6}$ & 2.29 & 6.07 & 5.55 & 1.92 & 4.72 & 5.48 \\
\hline $\mathbf{7}$ & 0.76 & 2.78 & 3.62 & 1.10 & 3.00 & 3.63 \\
\hline $\mathbf{8}$ & 5.69 & 5.70 & 2.81 & 5.55 & 5.75 & 3.64 \\
\hline Median & $\mathbf{9 . 4 6}$ & $\mathbf{6 . 6 2}$ & $\mathbf{4 . 9 0}$ & $\mathbf{9 . 5 5}$ & $\mathbf{6 . 5 8}$ & $\mathbf{5 . 0 5}$ \\
\hline
\end{tabular}

\section{Discussion}

We have constructed a proposed CFA based camera system using a normal $R G B$ and $R^{F} G^{F} B^{F}$ filters. The optimal filter used to construct the proposed system has been selected by setting acceptable threshold values for the color and the illuminant estimation errors. The proposed system allows us to capture a color constant RGB image and at the same time a 6-channel multispectral image. Therefore, this could be used as a normal digital RGB camera with the capability of color constancy, or as a spectral camera, or both, depending on the application need. 
The experimental results show that the proposed 6-channel multispectral system outperforms the 3-channel system both spectrally and colorimetrically. Moreover, the illuminant estimation with the chromagenic algorithm produces better results than the gray world and the max-RGB methods. We should note here that Finlayson et al. [12 have shown that the chromagenic based algorithms outperforms other color constancy algorithms, like neural network, LP gamut mapping, Bayesian method, and color by correlation. The results are, as expected, slightly poorer than the ideal system where there is no need of interpolation, but requiring three sensors instead of one. We have used it as the reference for comparing the results. It is important to note here that the results we obtained are from a simple bilinear interpolation for demosaicking. Bilinear interpolation introduces artifacts, especially around the edges, however the results are still promising. The results could be improved further with the use of a more sophisticated demosaicking algorithm.

The big challenge of the CFA based approach is the reduction in the spatial resolution due to the sparse distribution of the filters. We have used the CFA design that minimize the sparsity of the filters for the unfiltered RGB image as we can use it as a normal Bayer pattern, but with a reduced green. The reduction of one green channel might cause some reduction in information, and this could be one interesting study in the future to analyze how much this impact in the quality of the image.

The experimental results infer that simply selecting an optimal filter from a set of available filters produces promising results not only in the spectral and color reproduction from the multispectral imaging but also in the illuminant estimation. The performance could be improved further by using a set of a larger number of filters, possibly from different manufacturers. The performance could also be improved significantly by using a custom designed filter [13].

\section{Conclusion}

This paper proposes a CFA based camera system which is not only capable of acquiring a normal RGB image as well as a 6-channel multispectral image in a single shot, but also capable of estimating the illuminant under which the image is acquired. An optimal filter could either be selected from a set of available filers or custom designed. The system allows a user flexibility to capture a color constant RGB image or a multispectral image or both. The system could, therefore, be useful in many color imaging applications and computer vision.

An automatic filter selection method could be developed as a further work to this paper. The RGB image is obtained with one less green pixel compared to the classic Bayer's pattern. Detailed study can be made on its effect on the quality of the RGB image. 


\section{References}

1. Arend, L.E., Reeves, A., Schirillo, J., Goldstein, R.: Simultaneous color constancy: papers with diverse Munsell values. J. Opt. Soc. Am. A 8(4), 661-672 (1991)

2. Baone, G.A., Qi, H.: Demosaicking methods for multispectral cameras using mosaic focal plane array technology. In: Spectral Imaging: Eighth International Symposium on Multispectral Color Science. SPIE Proceedings, vol. 6062, pp. 75-87 (2000)

3. Barnard, K., Cardei, V.C., Funt, B.: A comparison of computational color constancy algorithms. i: Methodology and experiments with synthesized data. IEEE Transactions on Image Processing 11(9), 972-984 (2002)

4. Bayer, B.E.: Color imaging array (July 1976)

5. Brainard, D.H., Kraft, J.M., Longere, P.: Color constancy: developing empirical tests of computational models. In: Mausfeld, R., Heyer, D. (eds.) Colour Perception: From Light to Object, pp. 307-334. Oxford University Press (2003)

6. Brauers, J., Aach, T.: A color filter array based multispectral camera. In: Group, G.C. (ed.) 12. Workshop Farbbildverarbeitung. Ilmenau (October 2006)

7. Buchsbaum, G.: A spatial processor model for object colour perception. Journal of the Franklin Institute 310(1), 1-26 (1980)

8. Cardei, V.C., Funt, B., Barnard, K.: Estimating the scene illumination chromaticity by using a neural network. J. Opt. Soc. Am. A 19(12), 2374-2386 (2002)

9. Connah, D.R., Hardeberg, J.Y.: Spectral recovery using polynomial models. In: Color Imaging X: Processing, Hardcopy, and Applications. SPIE Proceedings, vol. 5667, pp. 65-75 (2005)

10. Farrell, J.E., Sherman, D., Wandell, B.: How to turn your scanner into a colorimeter. In: Tenth International Congress on Advances in Non-Impact Printing Technologies, pp. 579-581 (1994)

11. Finlayson, G.D., Hordley, S.D., HubeL, P.M.: Color by correlation: a simple, unifying framework for color constancy. IEEE Transactions on Pattern Analysis and Machine Intelligence 23(11), 1209-1221 (2001)

12. Finlayson, G.D., Hordley, S.D., Morovic, P.: Chromagenic colour constancy. In: 10th Congress of the International Colour Association (AIC), Granada, Spain, pp. 8-13 (May 2005)

13. Finlayson, G.D., Hordley, S.D., Morovic, P.: Chromagenic filter design. In: 10th Congress of the International Colour Association (AIC), Granada, Spain, pp. 10791083 (May 2005)

14. Forsyth, D.A.: A novel algorithm for color constancy. Int. J. Comput. Vision 5, 5-36 (1990)

15. Fredembach, C., Finlayson, G.D.: The bright-chromagenic algorithm for illuminant estimation. Journal of Imaging Science and Technology 52(4), 040906-1-040908-11 (2008)

16. Gehler, P.V., Rother, C., Blake, A., Minka, T., Sharp, T.: Bayesian color constancy revisited. In: IEEE Computer Society Conference on Computer Vision and Pattern Recognition (CVPR), Anchorage, Alaska, USA, pp. 1-8 (June 2008)

17. Hardeberg, J.Y., Schmitt, F., Brettel, H.: Multispectral color image capture using a liquid crystal tunable filter. Optical Engineering 41(10), 2532-2548 (2002)

18. Hardeberg, J.Y.: Filter selection for multispectral color image acquisition. Journal of Imaging Science and Technology 48(2), 105-110 (2004)

19. Hordley, S.D., Finlayson, G.D.: Reevaluation of color constancy algorithm performance. J. Opt. Soc. Am. A 23(5), 1008-1020 (2006) 
20. Huang, H.H.: Acquisition of multispectral images using digital cameras. In: Asian Association on Remote Sensing (ACRS) (2004)

21. Imai, F.H., Berns, R.S.: Spectral estimation using trichromatic digital cameras. In: International Symposium on Multispectral Imaging and Color Reproduction for Digital Archives, pp. 42-49 (1999)

22. Land, E.H.: The retinex theory of color vision. Scientific American 237(6), 108-128 (1977)

23. Lu, Y.M., Fredembach, C., Vetterli, M., Susstrunk, S.: Designing color filter arrays for the joint capture of visible and near-infrared images. In: IEEE Proceedings of the International Conference on Image Processing (2009)

24. Mansouri, A.M., Marzani, F.S., Gouton, P.: Neural networks in two cascade algorithms for spectral reflectance reconstruction. In: IEEE International Conference on Image Processing, pp. 2053-2056 (2005)

25. Miao, L., Qi, H.: A generic method for generating multi-spectral filter array. In: IEEE Proceedings of the International Conference on Image Processing, pp. 33433346 (2004)

26. Miao, L., Qi, H., Ramanath, R., Snyder, W.: Binary tree-based generic demosaicking algorithm for multispectral filter arrays. IEEE Transactions on Image Processing 15(11), 3550-3558 (2006)

27. Nascimento, S.M.C., Ferreira, F.P., Foster, D.H.: Statistics of spatial coneexcitation ratios in natural scenes. J. Opt. Soc. Am. A 19(8), 1484-1490 (2002)

28. Omega: Omega filters. Omega Optical, Inc., https://www.omegafilters.com/ Products/Curvomatic (Last Visited: November 2012)

29. Shrestha, R., Hardeberg, J.Y., Mansouri, A.: One-shot multispectral color imaging with a stereo camera. In: Imai, F.H., Xiao, F. (eds.) Digital Photography VII, Electronic Imaging. Proceedings of SPIE/IS\&T Electronic Imaging, vol. 7876, pp. 787609-787609-11. SPIE, San Francisco (2011)

30. Shrestha, R., Hardeberg, J.Y.: Computaional color constancy using a stereo camera. In: 6th European Conference on Color in Graphics, Image and Vision (CGIV). IS\&T, Amsterdam (May 2012)

31. Shrestha, R., Hardeberg, J.Y.: Computational color constancy using chromagenic filters in color filter arrays. In: Widenhorn, R., Nguyen, V., Dupret, A. (eds.) Sensors, Cameras, and Systems for Industrial/Scientific Applications XIII. Proceedings of SPIE/IS\&T Electronic Imaging, vol. 8298, pp. 82980S-82980S-9. SPIE, San Francisco (2012)

32. Shrestha, R., Hardeberg, J.Y.: Simultaneous Multispectral Imaging and Illuminant Estimation Using a Stereo Camera. In: Elmoataz, A., Mammass, D., Lezoray, O., Nouboud, F., Aboutajdine, D. (eds.) ICISP 2012. LNCS, vol. 7340, pp. 45-55. Springer, Heidelberg (2012)

33. Shrestha, R., Hardeberg, J.Y., Khan, R.: Spatial arrangement of color filter array for multispectral image acquisition. In: Widenhorn, R., Nguyen, V., Dupret, A. (eds.) Sensors, Cameras, and Systems for Industrial, Scientific, and Consumer Applications XII, Electronic Imaging. Proceedings of SPIE/IS\&T Electronic Imaging, vol. 7875, pp. 787503-787503-9. SPIE, San Francisco (2011)

34. Shrestha, R., Mansouri, A., Hardeberg, J.Y.: Multispectral imaging using a stereo camera: Concept, design and assessment. EURASIP Journal on Advances in Signal Processing 2011(1) (September 2011)

35. Tominaga, S.: Spectral imaging by a multichannel camera. Journal of Electronic Imaging 8(4), 332-341 (1999)

36. Yamaguchi, M., Haneishi, H., Ohyama, N.: Beyond Red-Green-Blue (RGB): Spectrum-based color imaging technology. Journal of Imaging Science and Technology 52(1), 10201-1-10201-15 (2008) 